\title{
ON THE AGE ESTIMATION OF LBDS 53W091
}

\author{
SUKYOUNG YI \\ Center for Space Astrophysics, Yonsei University, Seoul 120-749, Korea; and California Institute of Technology, Mail Code 405-47, Pasadena, CA 91125; \\ yi@srl.caltech.edu
}

AND

Thomas M. Brown, Sara Heap, Ivan Hubeny, Wayne Landsman, Thierry Lanz, and Allen Sweigart Laboratory for Astronomy and Solar Physics, Code 681, NASA Goddard Space Flight Center, Greenbelt, MD 20771

Received 1999 March 23; accepted 1999 September 29

\begin{abstract}
The recent spectral analysis of LBDS 53W091 by Spinrad and his collaborators has suggested that this red galaxy at $z=1.552$ is at least $3.5 \mathrm{Gyr}$ old. This imposes an important constraint on cosmology, suggesting that this galaxy formed at $z \gtrsim 6.5$, assuming recent estimates of cosmological parameters. While their analysis was heavily focused on the use of some UV spectral breaks as age indicators, we have performed $\chi^{2}$ tests to the continuum of this galaxy using its UV spectrum and photometric data $(R, J, H$, and $K: 2000-9000 \AA$ in rest frame). We have used the updated Yi models that are based on the Yale tracks. We find it extremely difficult to reproduce such large age estimates, under the assumption of the most probable input parameters. Using the same configuration as in Spinrad et al. (conventional solar abundance models), our analysis suggests an age of approximately 1.4-1.8 Gyr.

We have improved our models over conventional ones by taking into account convective core overshoot in the stellar model calculations and realistic metallicity distributions in the galaxy population synthesis. Overshoot affects the visible continuum normalized to the UV and raises the photometrybased age estimates by $25 \%$. The use of metallicity mixtures affects the whole spectrum and raises all continuum-based age estimates by up to a factor of 2 . If the mean metallicity of the stars in this galaxy is assumed to be twice solar, the models including these two effects match the UV spectrum and photometric data of LBDS 53W091 near the age of 1.5-2.0 Gyr. Our results cannot be easily reconciled with that of Spinrad et al. The discrepancy between Spinrad et al.'s age estimate (based on the Jimenez models) and ours originates from the large difference in the model integrated spectrum: the Jimenez models are much bluer than the Yi models and the Bruzual \& Charlot models. We propose to apply some viable tests to them for verification and search for the origin of the difference through a more thorough investigation. Considering the significance of the age estimates of distant galaxies as probes of cosmology, it would be an urgent task.
\end{abstract}

Subject headings: galaxies: elliptical and lenticular, $\mathrm{cD}$ - galaxies: evolution — galaxies: formation galaxies: individual (53W091) — galaxies: photometry — galaxies: stellar content

\section{INTRODUCTION}

Precise age estimates of high-redshift $(z)$ galaxies directly constrain the epoch of galaxy formation, $z_{f}$, where $z_{f}$ is defined as the epoch when the majority of stars formed. Constraining $z_{f}$ is important to cosmology. For example, one of the key questions in modern cosmology has been whether the majority of stars in giant elliptical galaxies form at high redshifts through violent starbursts or during rather recent merger/interaction activities between smaller galaxies. In addition, the age of a galaxy is a unique product of just a few cosmological parameters (e.g., $\Omega, \Lambda, H_{0}, z_{f}$ ); thus, it can be used to constrain cosmological parameters as well.

Spinrad and his collaborators recently obtained the restframe UV spectrum of LBDS 53W091, a very red galaxy at $z=1.552$, using the Keck Telescope (Dunlop et al. 1996; Spinrad et al. 1997). Based on their analysis on the UV spectrum and $R-K$ color, they have concluded that LBDS 53W091 is at least $3.5 \mathrm{Gyr}$ old already at $z=1.552$, which suggests $H_{0}<45 \mathrm{~km} \mathrm{~s}^{-1} \mathrm{Mpc}^{-1}$, in the context of the Einstein-de Sitter cosmology. When more recently measured cosmological parameters are used (e.g., $H_{0}=65$, $\Omega=0.3, \Lambda=0.7$; Aldering et al. 1998), this age estimate suggests that LBDS 53W091 formed at $z_{f} \gtrsim 6.5$.

Spinrad et al.'s results have been disputed by two independent studies. Bruzual \& Magris (1997a) combined the
UV spectrum studied by Spinrad et al. with $R, J, H, K$ photometry and obtained an age estimate 1.4 Gyr. Heap et al. (1998) interpreted the UV spectral breaks using model atmosphere results specifically constructed for their study and using the 1997 version Yale isochrones with no convective core overshoot (see $\S 3$ ). They have estimated that the age of LBDS 53W091 lies between 1 and 2 Gyr. They found that the Kurucz spectral library, which is currently used in virtually all population synthesis studies including that of Spinrad et al., does not match the detailed spectral features in the UV spectrum of an F-type main-sequence (MS) star obtained with the Hubble Space Telescope/Space Telescope Imaging Spectrograph (HST/STIS), in terms of the magnitudes of the spectral breaks used in the analysis of Spinrad et al. (1997). This implies that Spinrad et al.'s age estimate using UV spectral breaks may suffer from some systematic errors. Such discordant age estimates undermine our efforts to use this important technique as a probe of cosmology.

We have carried out a similar exercise, estimating the age of this galaxy, using the Yi population synthesis models ( $\mathrm{Yi}$, Demarque, \& Oemler 1997b). In this paper, we present the results from the UV-visible continuum analysis only. An analysis on the UV spectral breaks is currently in progress. We attempt to improve our age estimate by adopting (1) convective core overshoot in stellar model construction and 
(2) a realistic metallicity distribution, rather than the singleabundance assumption, in the galaxy population synthesis. We search for the sources of disagreement among various age estimates and provide a more reliable estimate.

Our analysis leads to an age estimate for LBDS 53W091 of approximately 1.5-2.0 Gyr, only half of Spinrad et al.'s estimate, but consistent with those of Bruzual \& Magris (1997a) and Heap et al. (1998). This smaller age estimate relaxes the strong constraint on $H_{0}$ and/or on $z_{f}$ implied by the Spinrad et al. estimate.

\section{MODEL CONSTRUCTION}

An important advantage of working with the spectra of giant elliptical galaxies at $z \approx 1-2$ comes from the fact that their major light sources in the UV and visible are all relatively well understood. This redshift range corresponds approximately to the age of 1-5 Gyr, depending on the cosmology adopted. Most of the UV light in 1-5 Gyr old stellar populations comes from stars near the mainsequence turn-off (MSTO). Because the mean temperature of MSTO stars in a coeval stellar population is a reliable indicator of the age of the population, the integrated UV spectra of such galaxies can provide direct clues to their ages. At such small ages, in fact, the whole spectrum is a reasonable age indicator, because the spectral evolution is rapid with time and the stellar evolution of all major light sources (the MS and the red giant branch [RGB]) is reasonably well understood. Thus, we have used both UV and visible data of LBDS 53W091 for the age estimation.

For the purposes of this population synthesis, we have updated the Yi models (Yi et al. 1997b) by using isochrones that are more carefully constructed than before. We have constructed stellar evolutionary tracks for the masses of 0.4-2.2 $M_{\odot}$ and the metallicities of $Z=0.005,0.02$, and 0.04 , using the Yale code. The tracks have been constructed with up-to-date input physics, including the 1995 version OPAL opacities (introduced in Rogers \& Iglesias 1992), as described in Yi, Demarque, \& Kim (1997a). Figure 1 shows a set of stellar evolutionary tracks for $Z=0.02$

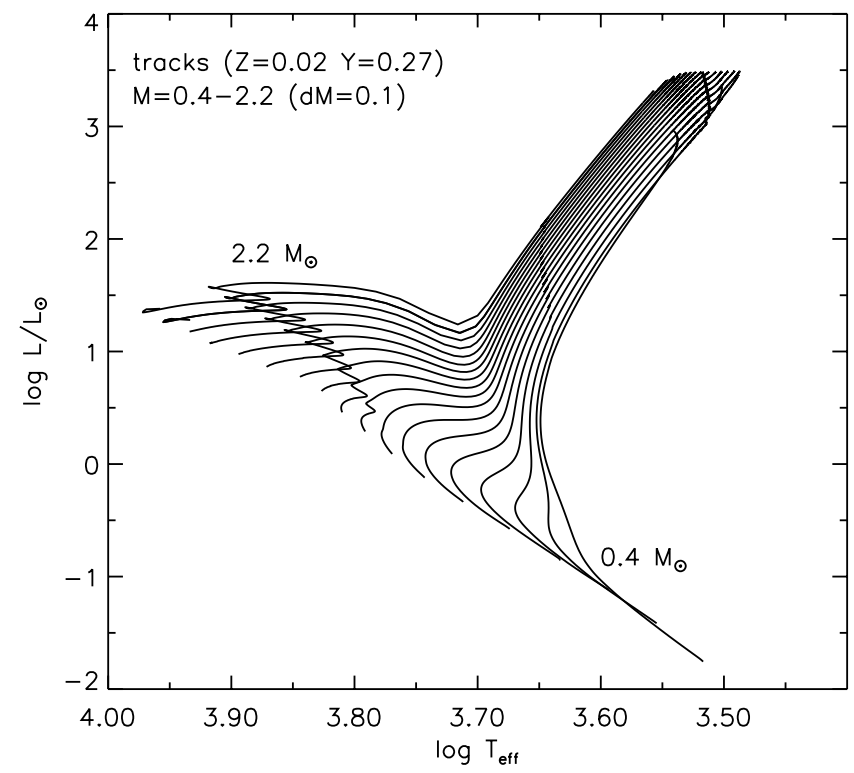

FIG. 1.- Stellar evolutionary tracks from the MS through RGB for $Z=0.02, Y=0.27$, and $M=0.4-2.2 M_{\odot}$. (approximately solar). The mean masses of MSTO stars in 1-5 Gyr, solar abundance populations range approximately from 2.2 to $1.3 M_{\odot}$.

It is not a simple task to construct isochrones from stellar evolutionary tracks, mainly because the interpolation between tracks is not trivial. Even if the same tracks are used, different isochrone routines may introduce notable disagreements. The mass interpolation must be carried out with particular caution. A small error in mass can cause a seriously inaccurate luminosity function near the tip of the RGB, which will in turn affect the integrated spectrum. For example, when 60 points define one isochrone from the zero-age MS through RGB, an error in mass in the fourth (when age $<5 \mathrm{Gyr}$ ) and fifth (age $\gtrsim 5 \mathrm{Gyr}$ ) digits below the decimal (in $M_{\odot}$ ) causes a noticeable difference in the integrated magnitudes in the near-infrared, but not necessarily in the shape of the isochrone.

The tip of the RGB is also difficult to locate, partly because it is somewhat sensitive to the adopted physics. Despite that, it is still important to define it as precisely and consistently as possible. Because the visible flux is dominated by bright red giants, an error in the position of the RGB tip leads to an error in the visible flux and thus in the normalized UV flux. The UV spectrum is relatively immune againt such complexities regarding the RGB construction, and thus one may argue that a UV spectrum is a better age indicator than the spectrum in longer wavelength regions.

Figure 2 shows a set of new isochrones constructed from the tracks shown in Figure 1, compared with those in the Revised Yale Isochrones (RYI; Green, Demarque, \& King 1987). The use of improved opacities and energy generation rates has led to a change in the shape of the isochrones. The RYI were designed mainly for isochrone fitting to the observed color-magnitude diagrams (CMDs) of globular clusters, and, thus, its creators did not pay much attention to the precise location of the RGB tips. As a result, it is not uncommon to see in the RYI some discontinuities in the brightness of RGB tips as a function of age, not to mention

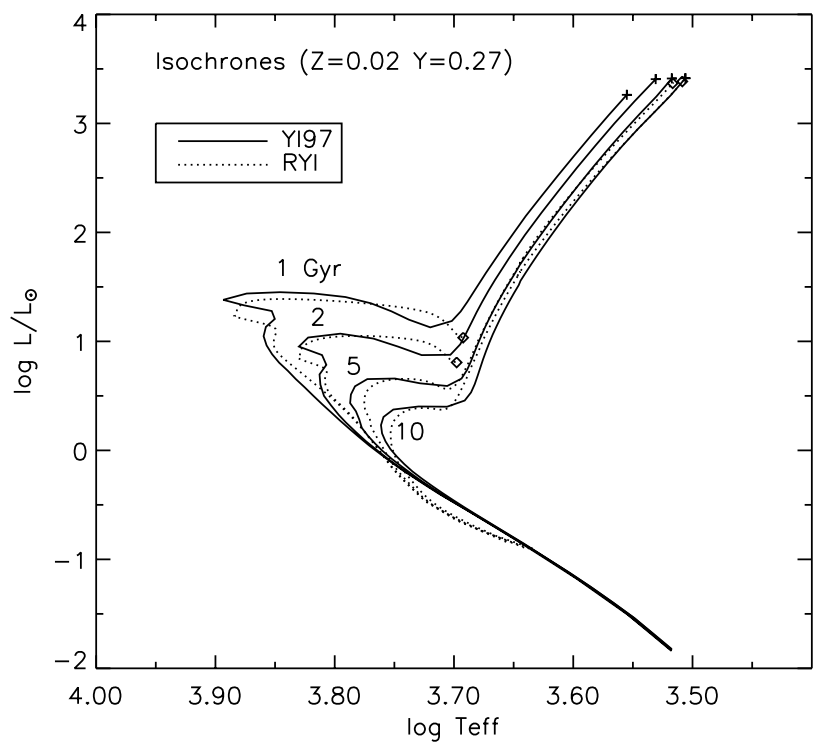

Fig. 2.-New Yale Isochrones for $Z=0.02, Y=0.27, t=1,2,5$, and 10 Gyr compared with the Revised Yale Isochrones (Green et al. 1987). The last point in each isochrone has been marked with diamonds and crosses. It is important to define the RGB tips carefully in the UV population synthesis. 
that some isochrones for small ages do not reach the RGB tip at all. The isochrones that are used in this study are shown in Figure 3. A sample is shown in Table 1. A complete set of the new Yale Isochrones will be published soon.

The post-RGB phase in the population synthesis, including the horizontal branch (HB), has been constructed following the description of Yi et al. (1997b). The adopted input parameters are $\eta=0.7$ (the mass-loss efficiency parameter in Reimers's empirical formula) and $\sigma_{\mathrm{HB}}=0.04$ $M_{\odot}$ (the HB mass-dispersion parameter). The justification for these choices is provided in Yi et al. (1999). However, details of the post-RGB prescriptions hardly affect the overall integrated spectrum at small ages, which is why it is particularly feasible to estimate the ages of giant elliptical galaxies at $z=1-2$. All models in this paper are based on the instantaneous starburst hypothesis.

Synthetic CMDs are then convolved with the Kurucz spectral library (Kurucz 1992) to generate integrated spectra. Figure 4 shows the integrated spectra of 1, 2, and 3 Gyr models for solar compositions compared to the observed data of LBDS 53W091. The rest-frame UV spectrum of LBDS 53W091 is from Spinrad et al. (1997).

The $R, J, H$, and $K$ magnitudes, obtained from the same source, have been converted into relative fluxes following equation (1):

$$
\begin{aligned}
\frac{F_{X}}{F_{3150}} & =\frac{F_{R}}{F_{3150}}\left[\frac{F_{X}}{F_{R}}\right]_{\mathrm{Vega}} 10^{0.4(R-X)} \\
& =0.414\left[\frac{F_{X}}{F_{R}}\right]_{\mathrm{Vega}} 10^{0.4(R-X)},
\end{aligned}
$$

where $F_{X}$ is the flux from an object of magnitude $X$ $(R=24.5 \pm 0.2, J=20.5 \pm 0.1, H=19.5 \pm 0.1, K=18.7$ $\pm 0.1)$ and $F_{3150}$ is the mean flux in the wavelength range 3000-3300 $\AA$. Vega's flux ratios have been computed from the Vega spectrum provided in the Kurucz library. They are $F_{J} / F_{R}=0.147, F_{H} / F_{R}=0.052$, and $F_{K} / F_{R}=0.019$. The resulting relative fluxes of LBDS 53W091 are $F_{R} / F_{3150}=$ $0.414, \quad F_{J} / F_{3150}=2.423, \quad F_{H} / F_{3150}=2.153, \quad$ and

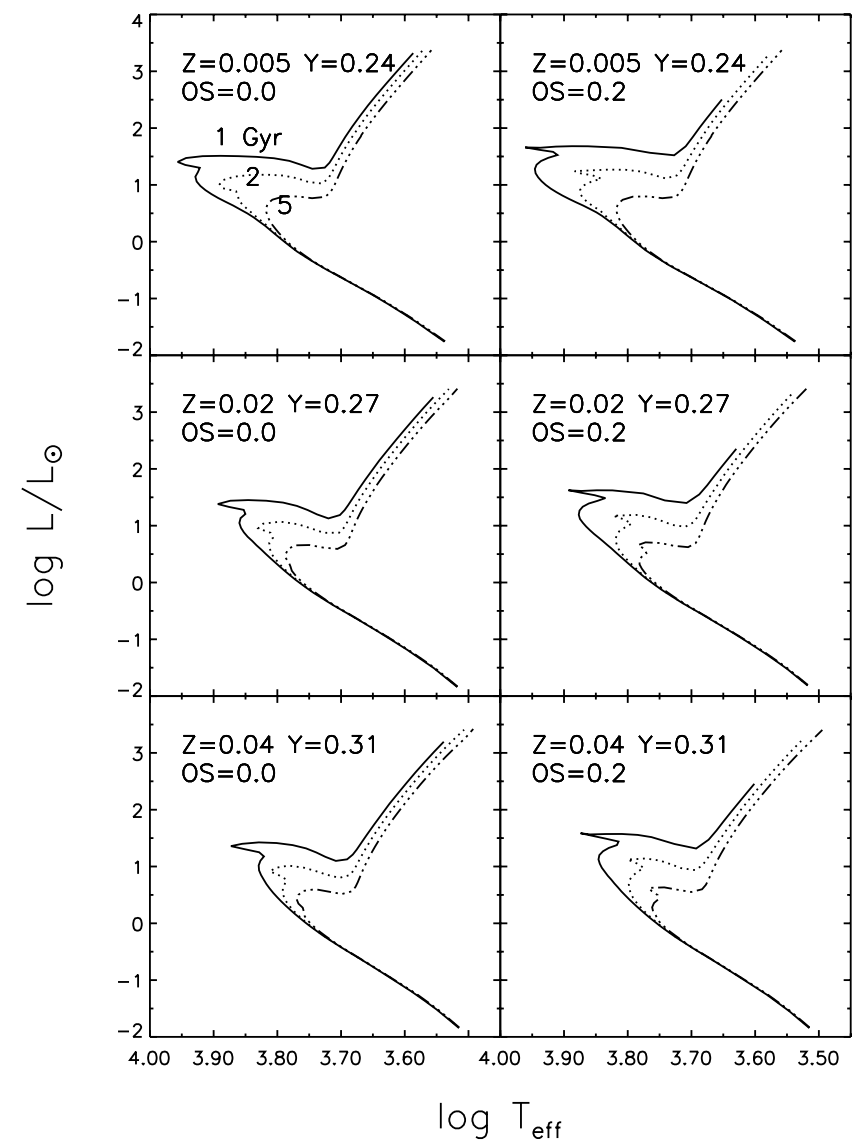

FIG. 3.-Part of the new Yale Isochrones that are used in this study. Each panel shows 1, 2, and $5 \mathrm{Gyr}$ isochrones with and without overshoot. These isochrones are available from this paper through the Astrophysical Journal.

$F_{K} / F_{3150}=1.643$, with approximately $20 \%, 10 \%, 10 \%$, and $10 \%$ observational uncertainties, respectively. Our relative fluxes in $R, J$, and $K$ agree with those in Bruzual \& Magris (1997a) within $1 \%-2 \%$ difference, but our $H$-band relative

TABLE 1

YALE ISOCHORNES 1997: SAMPLE FOR AGE $=1 \mathrm{GYR}, Z=0.02, Y=0.27$, AND OS $=0$

\begin{tabular}{ccc|ccc|ccc}
\hline \hline Mass $\left(M_{\odot}\right)$ & $\log T_{\text {eff }}$ & $\log L / L_{\odot}$ & Mass $\left(M_{\odot}\right)$ & $\log T_{\text {eff }}$ & $\log L / L_{\odot}$ & Mass $\left(M_{\odot}\right)$ & $\log T_{\text {eff }}$ & $\log L / L_{\odot}$ \\
\hline $0.4000000 \ldots \ldots$ & 3.5176 & -1.8407 & $1.2575005 \ldots \ldots$ & 3.8032 & 0.3505 & $2.1174183 \ldots \ldots$ & 3.7525 & 1.2761 \\
$0.4512092 \ldots \ldots$ & 3.5360 & -1.6738 & $1.2997627 \ldots \ldots$ & 3.8108 & 0.4266 & $2.1190669 \ldots \ldots$ & 3.7377 & 1.1991 \\
$0.5031496 \ldots \ldots$ & 3.5544 & -1.5073 & $1.3475087 \ldots \ldots$ & 3.8181 & 0.5025 & $2.1214807 \ldots \ldots$ & 3.7199 & 1.1285 \\
$0.5549585 \ldots \ldots$ & 3.5755 & -1.3443 & $1.3959177 \ldots \ldots$ & 3.8252 & 0.5790 & $2.1259794 \ldots \ldots$ & 3.7018 & 1.1871 \\
$0.6073884 \ldots \ldots$ & 3.5966 & -1.1818 & $1.4484234 \ldots \ldots$ & 3.8326 & 0.6539 & $2.1291104 \ldots \ldots$ & 3.6944 & 1.2742 \\
$0.6617560 \ldots \ldots$ & 3.6192 & -1.0226 & $1.5025389 \ldots \ldots$ & 3.8393 & 0.7301 & $2.1321655 \ldots \ldots$ & 3.6888 & 1.3627 \\
$0.7174781 \ldots \ldots$ & 3.6419 & -0.8641 & $1.5604186 \ldots \ldots$ & 3.8466 & 0.8048 & $2.1354712 \ldots \ldots$ & 3.6836 & 1.4514 \\
$0.7759390 \ldots \ldots$ & 3.6651 & -0.7079 & $1.6214427 \ldots \ldots$ & 3.8525 & 0.8823 & $2.1390638 \ldots \ldots$ & 3.6784 & 1.5400 \\
$0.8362966 \ldots \ldots$ & 3.6886 & -0.5529 & $1.6869364 \ldots \ldots$ & 3.8571 & 0.9627 & $2.1429518 \ldots \ldots$ & 3.6732 & 1.6286 \\
$0.8973278 \ldots \ldots$ & 3.7125 & -0.3990 & $1.7595468 \ldots \ldots$ & 3.8596 & 1.0476 & $2.1429518 \ldots \ldots$ & 3.6732 & 1.6286 \\
$0.8973278 \ldots \ldots$ & 3.7125 & -0.3990 & $1.8357059 \ldots \ldots$ & 3.8577 & 1.1327 & $2.1514939 \ldots \ldots$ & 3.6616 & 1.8154 \\
$0.9292600 \ldots \ldots$ & 3.7230 & -0.3257 & $1.9051671 \ldots \ldots$ & 3.8502 & 1.2060 & $2.1593514 \ldots \ldots$ & 3.6495 & 2.0018 \\
$0.9612948 \ldots \ldots$ & 3.7333 & -0.2522 & $1.9590699 \ldots \ldots$ & 3.8528 & 1.2785 & $2.1689492 \ldots \ldots$ & 3.6398 & 2.1507 \\
$0.9931888 \ldots \ldots$ & 3.7436 & -0.1786 & $1.9677832 \ldots \ldots$ & 3.8759 & 1.3307 & $2.1730153 \ldots \ldots$ & 3.6264 & 2.3366 \\
$1.0279125 \ldots \ldots$ & 3.7531 & -0.1043 & $1.9703208 \ldots \ldots$ & 3.8935 & 1.3813 & $2.1761145 \ldots \ldots$ & 3.6128 & 2.5223 \\
$1.0633708 \ldots \ldots$ & 3.7623 & -0.0289 & $1.9929478 \ldots \ldots$ & 3.8738 & 1.4393 & $2.1784258 \ldots \ldots$ & 3.5989 & 2.7079 \\
$1.0986109 \ldots \ldots$ & 3.7713 & 0.0461 & $2.1043307 \ldots \ldots$ & 3.8458 & 1.4514 & $2.1801612 \ldots \ldots$ & 3.5847 & 2.8932 \\
$1.1368794 \ldots \ldots$ & 3.7796 & 0.1219 & $2.1094252 \ldots \ldots$ & 3.8175 & 1.4395 & $2.1814793 \ldots \ldots$ & 3.5701 & 3.0782 \\
$1.1753701 \ldots \ldots$ & 3.7877 & 0.1980 & $2.1129504 \ldots \ldots$ & 3.7910 & 1.4071 & $2.1849382 \ldots \ldots$ & 3.5551 & 3.2624 \\
$1.2151288 \ldots \ldots$ & 3.7956 & 0.2743 & $2.1155551 \ldots \ldots$ & 3.7694 & 1.3488 & & & \\
\hline
\end{tabular}



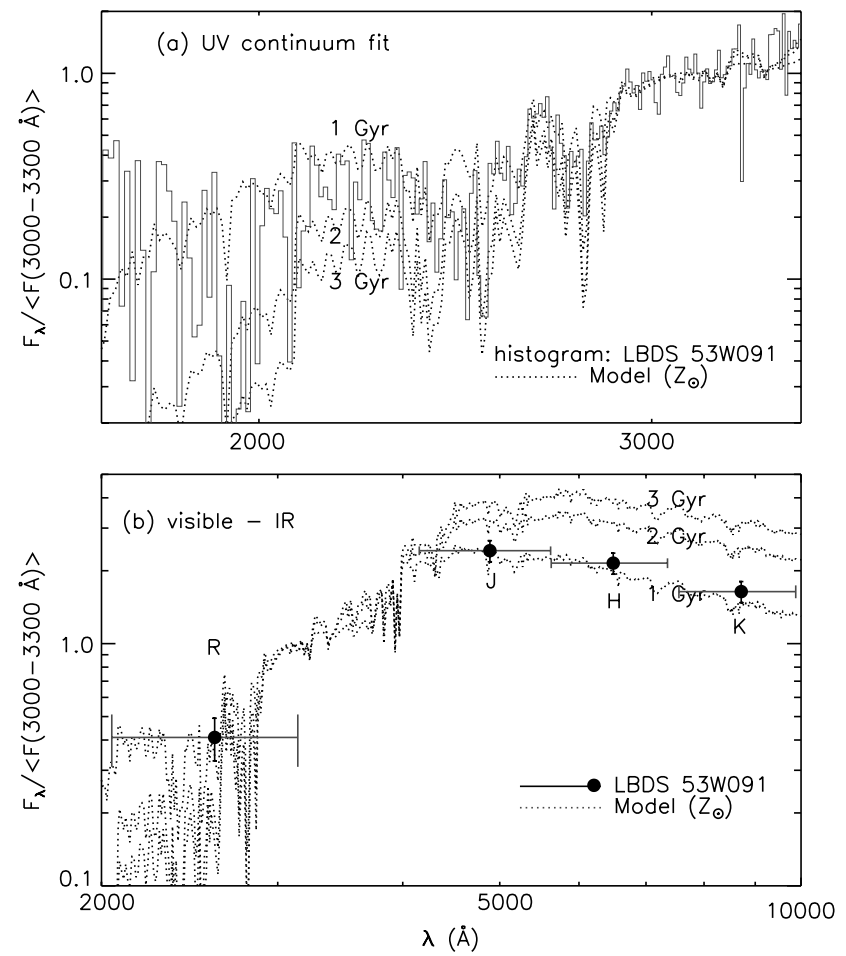

FIG. 4.- Model integrated spectra for solar abundance vs. the observed data of LBDS 53W091. The $x$-axis shows the rest-frame wavelength. All data of LBDS 53W091 are from Spinrad et al. (1997). The four photometric data (filled circles) have been derived from their $R, J, H$, and $K$ magnitudes. The $y$-axis error bars are observational errors, and the $x$-axis ones show the effective bandwidths. The whole continuum is best matched if LBDS 53W091 is 1-2 Gyr old, when solar abundance is assumed.

flux is about $10 \%$ lower than theirs. However, this does not make an appreciable difference in the age estimate.

Whether we can simply combine these two sets of different data (the UV spectrum and the photometric data) may

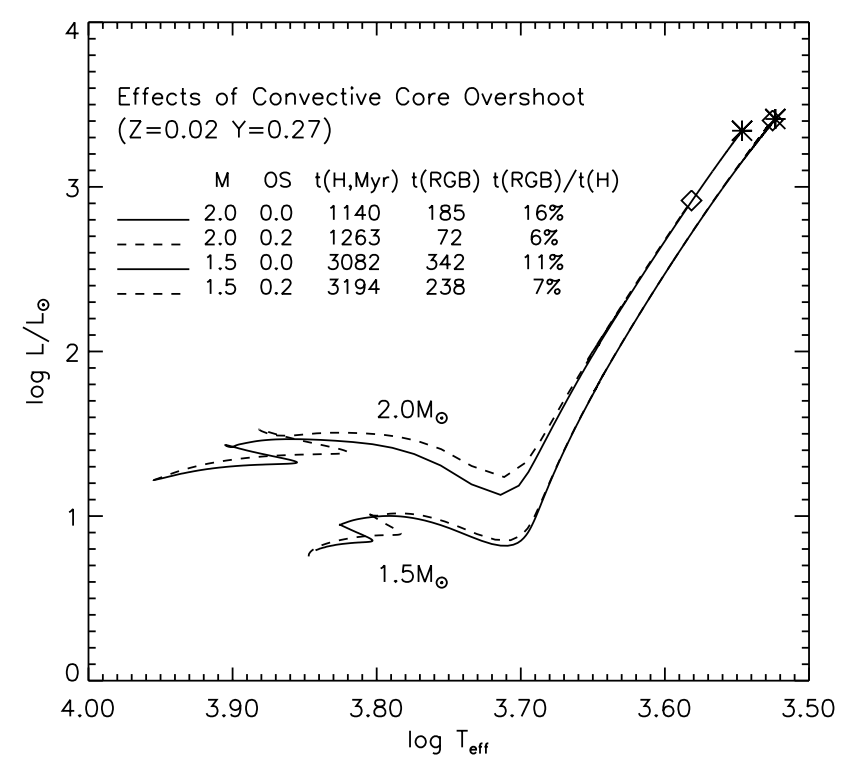

FIG. 5.-Effects of convective core overshoot (OS) to the stellar evolution tracks of 1.5 and $2.0 M_{\odot}$. An increase in the adopted amount of OS causes a longer lifetime in the hydrogen burning phase relative to that in the helium burning phase and a longer lifetime in the MS than in the RGB. The RGB tips are marked with diamonds (with OS) and asterisks (without OS).

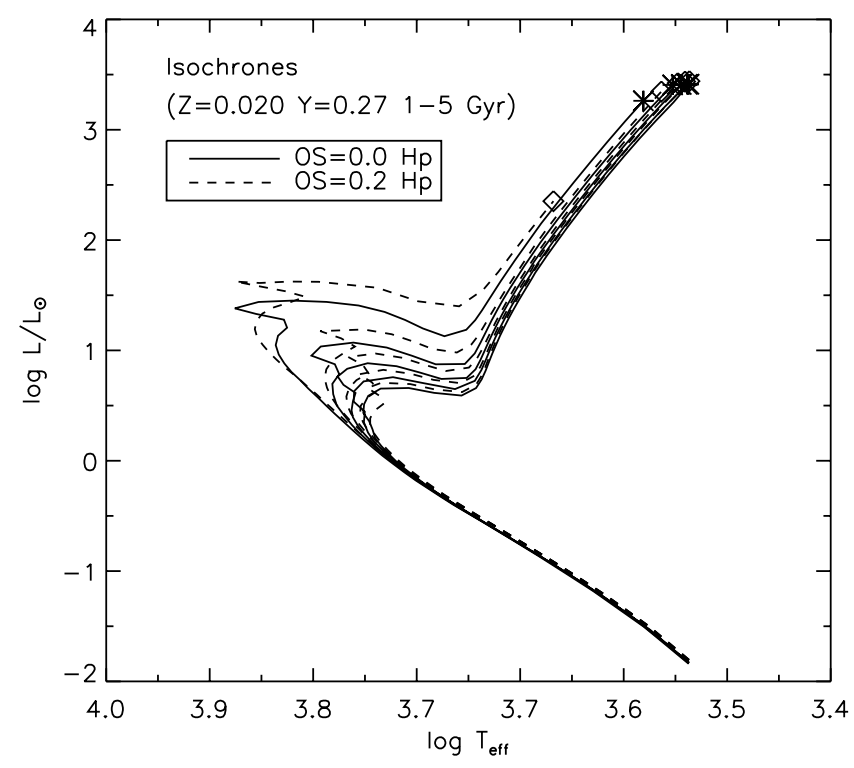

FIG. 6.-Isochrones with and without overshoot (OS). The tip of the RGB has been marked with diamonds (OS) and asterisks (no-OS). Note that the $1 \mathrm{Gyr}$ isochrone with OS does not extend as far as its counterpart (without OS) does: the RGB tip in the $1 \mathrm{Gyr}$ isochrone with OS is much fainter than that without OS. This causes a large difference in the integrated spectrum, as shown in Fig. 7.

be questionable because photometry covered a larger area of the galaxy than spectroscopy did (Spinrad et al. 1997). We will discuss this effect in $\S 5.3$. Despite that, both the UV spectrum and the photometric data indicate small ages between 1 and 2 Gyr consistently.
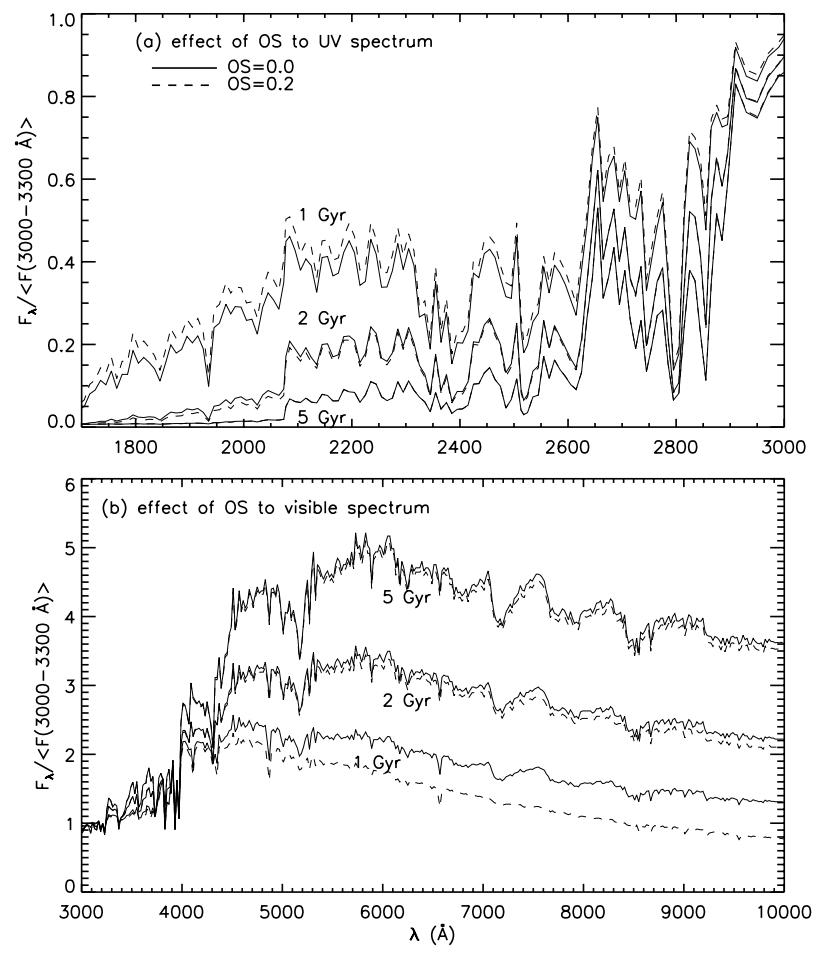

FIG. 7.-Model integrated spectra with and without overshoot (OS). Note that the effect of OS is appreciable only in the young ( $\$ 2 \mathrm{Gyr}$ ) model's visible-IR spectrum. 


\section{EFFECTS OF CONVECTIVE CORE OVERSHOOT}

Convective core overshoot (OS), the importance of which was first pointed out by Shaviv \& Salpeter (1973), is the inertia-induced penetrative motion of convective cells, reaching beyond the convective core as defined by the classic Schwarzschild (1906) criterion. Stars develop convective cores if their masses are larger than approximately 1.3-1.5 $M_{\odot}$, typical for the MSTO stars in 1-5 Gyr old populations, such as LBDS 53W091.

There has been a consensus for the presence of OS, but its extent has been controversial. Thus, conventional stellar models often do not include OS. Since the advent of the OPAL opacities, a major improvement in the stellar astrophysics, various studies have suggested a modest amount of $\mathrm{OS}$; that is, $\mathrm{OS} \approx 0.2 H_{p}$, where $H_{p}$ is the pressure scale height (Stothers 1991; Demarque, Sarajedini, \& Guo 1994; Kozhurina-Platais et al. 1997).

OS has many effects on stellar evolution, but the most notable ones are its effects on the shape of the MSTO and on the luminosity function. As shown in Figure 5, inclusion of a modest amount of OS causes a longer stretch of the MS before the blueward motion because it induces a larger supply of hydrogen fuel from the overshooting ("overmixing") region into the core. As a result, stars with OS $\left(=0.2 H_{p}\right)$ stay longer in the core hydrogen burning stage, but in the MS rather than in the RGB. The table in Figure 5 lists the lifetime in the hydrogen burning phase and in the RGB, both in Myr.

OS also causes stars to leave the RGB earlier. The diamonds in Figure 5 are the locations of the RGB tips when OS is included, while asterisks are for the no-OS case. This directly affects isochrones, as shown in Figure 6. The RGB tips in the isochrones reach farther without OS. Because most of the visible flux comes from red giants, a decrease in the RGB lifetime results in a lower visible flux. Figure 7 shows the integrated spectra with and without OS. One can see the impact of OS on the normalized integrated spectra of $1 \mathrm{Gyr}$ models and thus on the age estimates. When the isochrones with OS are used in the population synthesis, the same observed integrated spectrum indicates a larger age, especially when the age is as small as $1 \mathrm{Gyr}$.

\section{EFFECTS OF METALLICITY MIXTURE}

Most of the previous age estimates of LBDS 53W091 were based on the single-abundance population models, typically for solar composition. The solar abundance approximation has been popular for decades because reliable spectral libraries were available only for solar compositions. The first obvious problem in this approximation is that there is little justification in the choice of solar composition in modeling giant elliptical galaxies. Observers have long believed that the majority of stars in giant elliptical galaxies are metal rich (approximately twice solar) because of their extremely red colors and strong absorption lines. Moreover, most chemical evolution theories predict that giant elliptical galaxies would reach the current metallicity level within a few tenths of a Gyr of the initial starburst (e.g., Kodama \& Arimoto 1997). This means that 1-5 Gyr old giant elliptical galaxies may already have stars of various metallicities ranging $Z \approx 0$ through perhaps 3-4 times solar. Then, whether the single-abundance approximation would be appropriate for modeling giant elliptical galaxies is questionable.
Another problem is that the age estimate based on continuum fits is quite sensitive to the metallicity adopted. For example, if we assume a high metallicity for LBDS 53W091, the observed data of LBDS 53W091 would indicate an age smaller than 1 Gyr (Fig. 8c). On the other hand, if the majority of stars in the galaxies at such high redshifts were metal poor, the same data would indicate a much larger age by a factor of $2-3$ (Fig. 8a). This difference is caused by the opacity effects that increase with increasing metallicity. As a result, the same observed spectrum can indicate significantly different ages when the true metallicity is unknown. With such a large uncertainty in age estimate, it would be extremely difficult to use the age estimates of distant galaxies to constrain cosmology.

The release of the improved Kurucz theoretical spectral library (Kurucz 1992) has finally enabled realistic modeling for compositions other than solar. Thus, it is our intention to construct more realistic models than simplified solar abundance models. One can guess the effect of the use of metallicity mixtures even with a cursory inspection of Figure 8 . The fraction of metal-poor stars in conventional metallicity mixture models is small. However, even with such small fractions of metal-poor stars, a combination of coeval stars of various metallicities may have a substantial UV light contribution from metal-poor stars because of the opacity effects.

It should be noted that, while we try to make our models more realisitic than single-abundance models by adopting

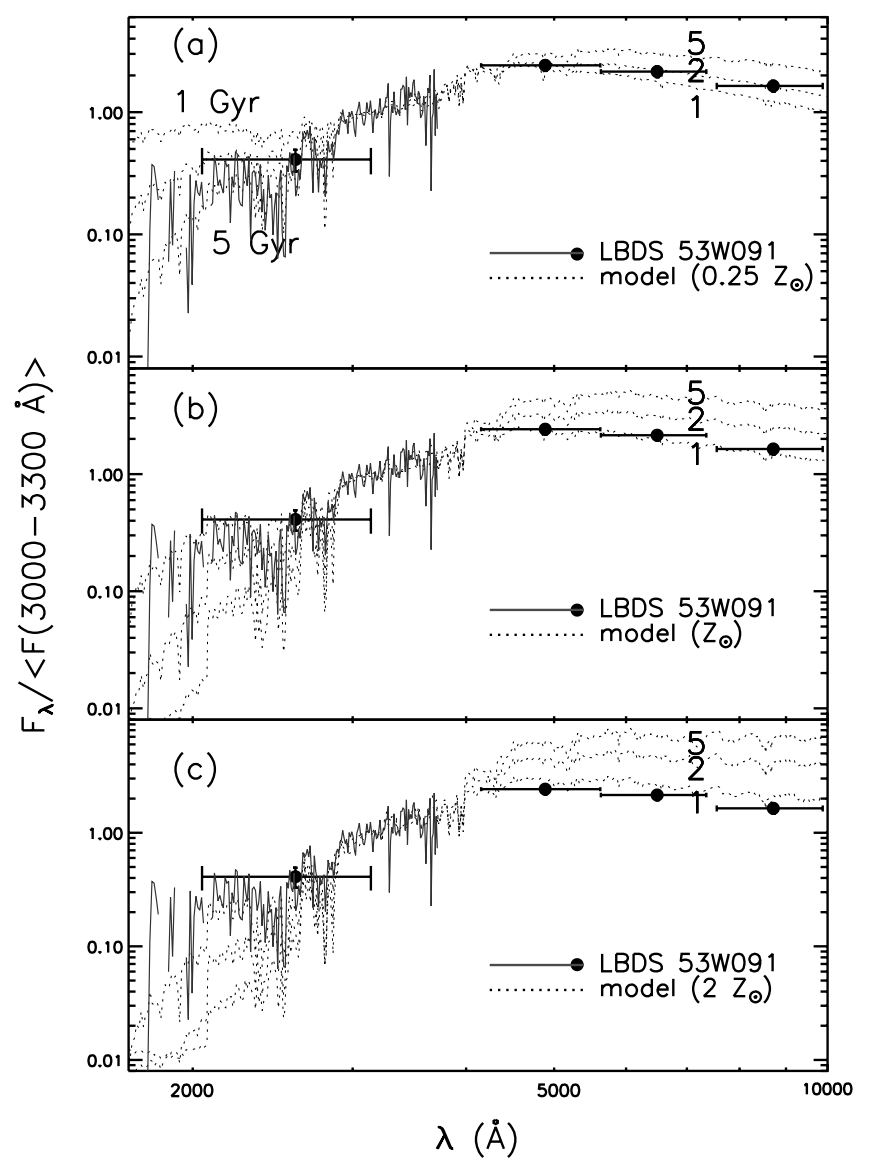

FIG. 8.-The effects of metallicity to the integrated spectrum. Models are for 1, 2, and $5 \mathrm{Gyr}$ and for no overshoot. An error in the adopted metallicity leads to a large error in the age estimate. One must adopt a realistic metallicity or metallicity distribution. 
metallicity mixtures and the Kurucz library, our models are still subject to the uncertainty in the spectral library. For example, Heap et al. (1998) noted that the Kurucz library does not reproduce the correlations between some UV spectral strengths and optical colors found in the International Ultraviolet Explorer sample. It is not our intention to claim that our age estimates based on composite models are free from such uncertainties in the spectral library. We only believe that the use of physically plausible metallicity mixtures would result in the models superior to singleabundance models, other uncertainties remaining the same.

We have adopted the chemical evolution models of Kodama \& Arimoto (1997) that reasonably match the observed properties of present epoch giant elliptical galaxies. We have adopted both their metal-rich $\left(\langle Z\rangle \approx 0.04 \approx 2 Z_{\odot}\right)$ "simple" and "infall" models for LBDS 53W091. Their simple model is a closed-box model where the star formation timescale is $0.1 \mathrm{Gyr}$. The galactic wind epoch, where the supernova-driven thermal energy exceeds the binding energy, is $0.353 \mathrm{Gyr}$ from the beginning of the starburst. The infall model is based on the same configuration, except that the unprocessed gas in the outskirt falls into the core with the timescale of $0.1 \mathrm{Gyr}$ (see Kodama \& Arimoto 1997 for details). When only three metallicity bins $(Z=0.005,0.02,0.04)$ are used, the fractions of metallicity groups are approximately $18 \%$ for $Z=0.005$, $24 \%$ for $Z=0.02,58 \%$ for $Z=0.04$ in the "simple" model, and $12 \%$ for $Z=0.005,31 \%$ for $Z=0.02,57 \%$ for $Z=0.04$ in the "infall" model. If giant elliptical galaxies at $z=1-2$ had significantly different metallicity distributions from these models, our composite models should be used for illustration purpose only.

Figure 9 illustrates the likely light contributions from various metallicity groups of stars when the galaxy is 1.5 Gyr old. As shown in Figure 9a, the composite-"infall" model with OS matches the overall data of LBDS 53W091

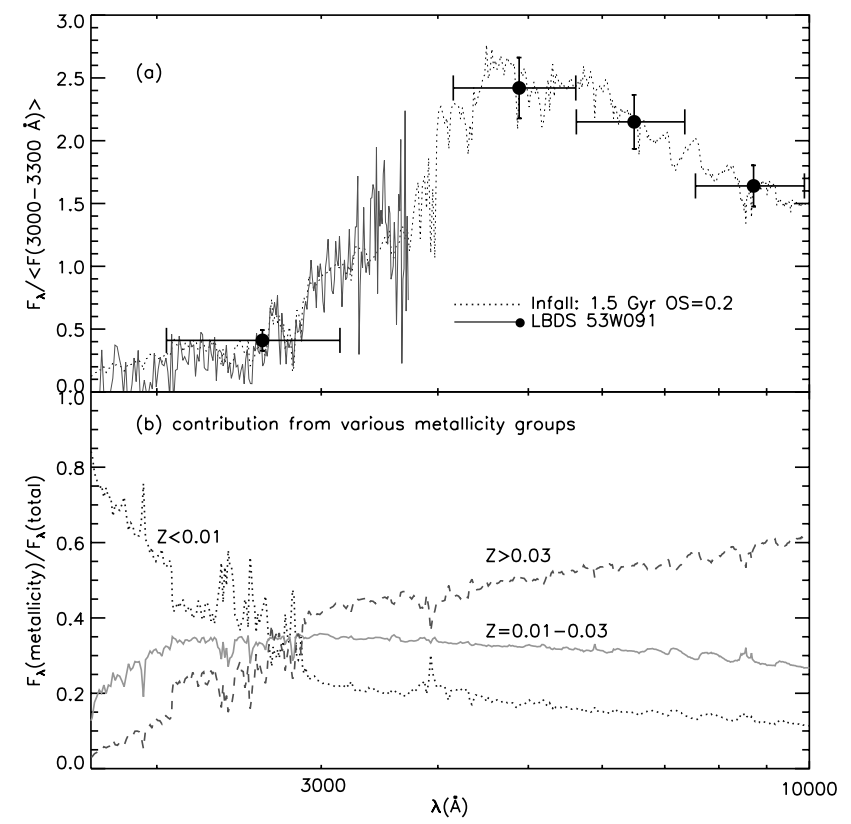

FIG. 9.-Top: Composite-infall model of $1.5 \mathrm{Gyr}$ of age fits the overall continuum of LBDS 53W091 reasonably well. Bottom: While the visible spectrum is dominated by metal-rich stars, the UV spectrum is strongly affected by metal-poor stars. Therefore, it is important to use a realistic metallicity distribution in the galaxy population synthesis. reasonably well at this age. As we expected, metal-poor stars are more efficient UV sources than metal-rich stars, while the opposite is true in the longer wavelength regions (Fig. 9b). The level of the light contribution from metal-poor stars is even higher when the "simple" (instead of "infall") model is used. This is because "simple" models generally predict a larger fraction of metal-poor stars than "infall" models do.

\section{RESULTS}

We perform $\chi^{2}$ minimization fits using the UV spectrum and the photometric data of LBDS 53W091, and the results are following.

\subsection{Fit to the UV Spectrum}

We have carried out a weighted $\chi^{2}$ test to the UV spectrum of LBDS 53W091, using the following definition,

$$
\chi^{2}=\frac{1}{N-2} \sum \frac{\left[f_{\lambda}(\text { model })-f_{\lambda}(\text { observed })\right]^{2}}{\sigma_{\lambda}^{2}},
$$

where $N$ and $\sigma_{\lambda}$ are the number of spectral bins and the observational errors, respectively.

Figure 10 shows the measured values of reduced $\chi^{2}$ of various models. A smaller value of $\chi^{2}$ indicates a better fit. The spectra are normalized at $3150 \AA$ (the average flux in the range of $3000-3300 \AA$ ), but the test was quite insensitive to the choice of the normalization point, as long as the normalization flux is the mean flux in a reasonably wide $(\Delta \lambda \gtrsim 50 \AA)$ range. We modified the error value at 4973.47

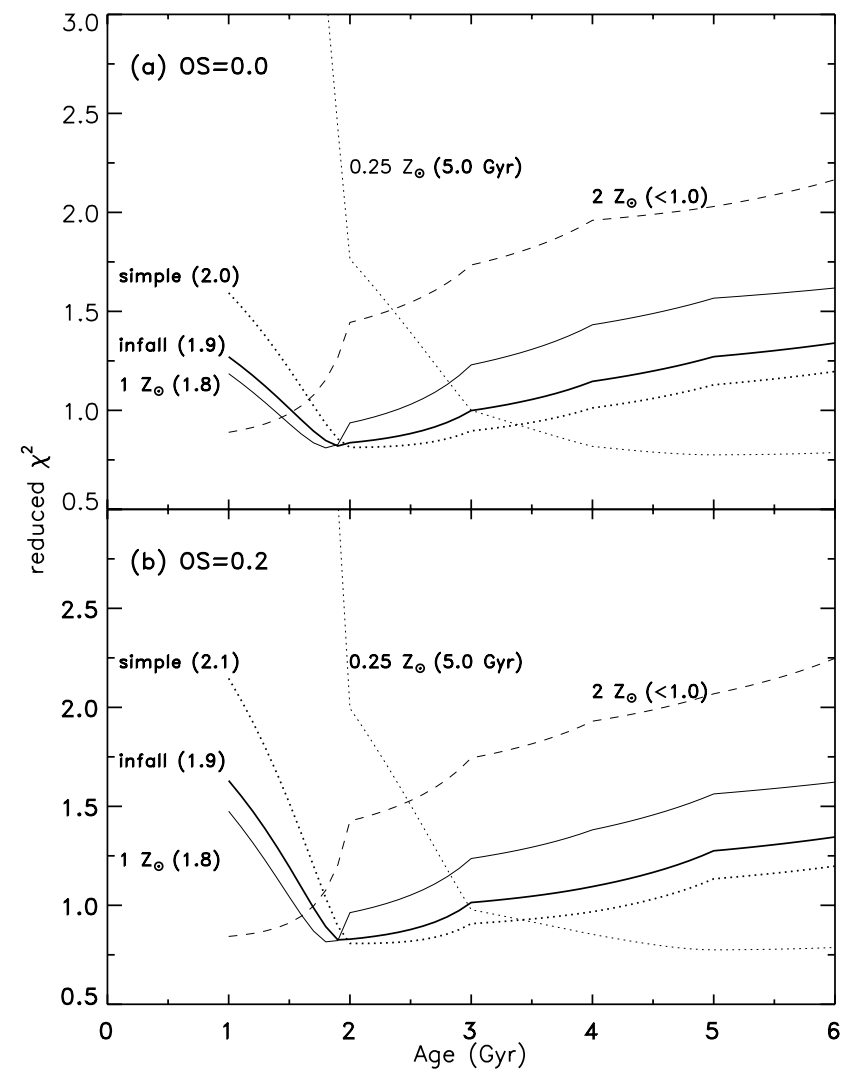

FIG. 10. - $\chi^{2}$ test of various models to the UV spectrum of LBDS 53W091. Each line is marked by the model name and the age of the model that shows the minimum $\chi^{2}$. Solar abundance models suggest 1.8-1.9 Gyr, and composite models suggest approximately 2 Gyr. Inclusion of overshoot has little impact. 
$\AA$ (1948.85 ̊ in the rest frame) with an approval from the observers. It was 2 orders of magnitude smaller than other values; thus $\chi^{2}$ tests were heavily dominated by the single point, producing unreasonable results. We assume that it was an artifact. We replaced it with an interpolated value from the adjacent bins.

Figure 10 shows that OS has very little effect on the age estimates based on the UV spectrum, as illustrated earlier in Figure 7. The discussion of the effects of metallicity mixtures requires a bit of clarification first. The mean metallicity of the majority of stars in giant elliptical galaxies is often believed to be approximately twice solar. If we use such a large metallicity for the single-abundance population synthesis, then we would tend to underestimate the UV flux of this galaxy significantly and thus the age $(<1.0 \mathrm{Gyr})$. This is because even in such metal-rich $\left(\langle Z\rangle \approx 2 Z_{\odot}\right)$ galaxies the main UV sources are probably metal poor (see Fig. 9). In this sense, the effect of the use of metallicity mixtures is very large. As one can see in Figure 10, solar abundance models mimic metal-rich $\left(2 Z_{\odot}\right)$ composite models reasonably. Composite models indicate larger ages (1.9-2.1 Gyr) than solar abundance models (1.8 Gyr) by approximately $10 \%$. This suggests that the solar abundance, used by various groups including Spinrad et al. (1997), might be a reasonable approximation in the UV population synthesis of young giant elliptical galaxies, even though it is still a factor of 2 smaller than the likely mean metallicity of stars in giant elliptical galaxies (cf. Angeletti \& Gianonne 1999). When the $3 \sigma$ confidence ranges are included, our age estimates based on the $\chi^{2}$ test using the UV spectrum alone are approximately $1.8_{-0.1}^{+0.2} \mathrm{Gyr}$ (solar abundance model with OS) through $1.9_{-0.1}^{+0.6}$ Gyr (infall model with OS), where the errors are internal only and do not include observational errors. Thus, they are inconsistent with that of Spinrad et al. (1997) with more than a $3 \sigma$ confidence $(99.7 \%)$.

\subsection{Fit to the Photometric Data}

Photometric data are available for LBDS 53W091 in $R$, $J, H$, and $K$ magnitudes, covering approximately 2000 $9000 \AA$ in the rest-frame wavelength at the redshift of this galaxy $(z=1.552)$. Photometric data may be less indicative of the age of a population than the UV spectrum, but it is also less affected by the uncertainties in reddening and in metallicity.

We have performed a weighted $\chi^{2}$ test on the four photometric data points. The errors in magnitudes were also adopted from Spinrad et al. (1997). Figure 11 shows the reduced $\chi^{2}$ as a function of age. The models with minimum $\chi^{2}$ indicate ages of less than 2 Gyr unless the majority of stars in LBDS 53W091 are very metal poor. Composite models with $\mathrm{OS}=0.2$ suggest ages of approximately 1.5 Gyr.

Unlike the analysis of the UV spectrum (§ 5.1), inclusion of OS raises the age estimates based on the photometric data substantially, $20 \%-50 \%$. This is because OS has a large impact on the visible-IR flux when normalized to the UV (see Fig. 7). If no OS is adopted, composite models suggest very small ages $(<1.0 \mathrm{Gyr})$. The effect of inclusion of metallicity mixtures is also substantial. When the mean metallicity of stars in LBDS 53W091 is assumed to be twice solar, our models with OS suggest $25 \%$ larger ages when metallicity mixtures are included. This is because composite models contain some metal-poor stars which match the

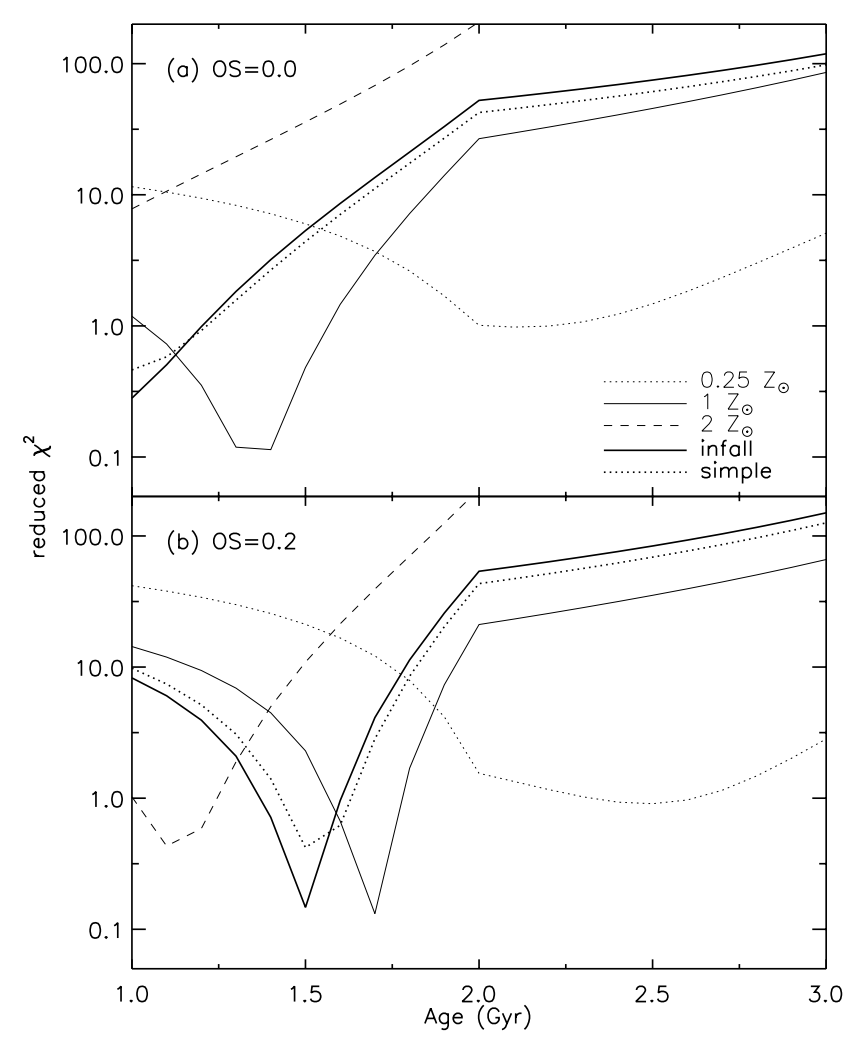

FIG. 11. $-\chi^{2}$ test of various models to the photometric data of LBDS 53W091. Composite models with OS, the most realistic models, reach their minimum $\chi^{2}$ near the age of $1.5 \mathrm{Gyr}$.

$R-J$ color (i.e., rest-frame UV continuum) at older ages than metal-rich stars do.

When we use composite models with OS, our analysis on the photometric data of LBDS 53W091 suggests $1.5 \pm 0.2$ Gyr, which is significantly smaller than the age estimate of Spinrad et al. but consistent with that of Bruzual \& Magris. This value is somewhat smaller than our estimate from the $\mathrm{UV}$ analysis in the previous section.

\subsection{The New Age Estimate of LBDS 53 W091}

Table 2 shows the list of our age estimates based on the two data sets. They range approximately 1-2 Gyr unless extreme metallicities are assumed. Figure 12 shows a

TABLE 2

Age Estimates (Gyr) OF LBDS 53W091 Based on $\chi^{2}$ Test $^{\mathrm{a}}$

\begin{tabular}{|c|c|c|c|}
\hline Metallicity & OS & $\begin{array}{l}\text { Estimate Using } \\
\text { UV Spectrum }\end{array}$ & $\begin{array}{l}\text { Estimate Using } \\
\text { Photometry }\end{array}$ \\
\hline $0.25 Z_{\odot} \ldots \ldots$ & 0.0 & $5.0_{-0.3}^{+0.6}$ & $2.1_{-0.2}^{+0.4}$ \\
\hline $0.25 Z_{\odot} \ldots \ldots$ & 0.2 & $5.0_{-0.2}^{+0.6}$ & $2.5_{-0.5}^{+0.3}$ \\
\hline$Z_{\odot} \ldots \ldots \ldots$ & 0.0 & $1.8 \pm 0.1$ & $1.4_{-0.3}^{+0.2}$ \\
\hline$Z_{\odot} \ldots \ldots \ldots \ldots$ & 0.2 & $1.8 \pm 0.1$ & $1.7 \pm 0.1$ \\
\hline $2 Z_{\odot} \ldots \ldots \ldots$ & 0.0 & $\lesssim 1.0$ & $\lesssim 1.0$ \\
\hline $2 Z_{\odot} \ldots \ldots \ldots$ & 0.2 & $\lesssim 1.0$ & $1.1_{-0.1}^{+0.2}$ \\
\hline Simple $^{b} \ldots \ldots$ & 0.0 & $2.0_{-0.1}^{+0.2}$ & $\lesssim 1.0$ \\
\hline Simple $^{b} \ldots \ldots$ & 0.2 & $2.1_{-0.2}^{+0.3}$ & $1.5_{-0.1}^{+0.2}$ \\
\hline Infall $^{\mathrm{B}} \ldots \ldots \ldots$ & 0.0 & $1.9 \pm 0.1$ & $\lesssim 1.0$ \\
\hline Infall $^{\mathrm{b}} \ldots \ldots \ldots$ & 0.2 & $1.9_{-0.1}^{+0.2}$ & $1.5 \pm 0.1$ \\
\hline
\end{tabular}

${ }^{a}$ Errors are systematic errors for $1 \sigma$. The resolution of our models in age is $0.1 \mathrm{Gyr}$.

${ }^{\mathrm{b}}$ Composite models from Kodama \& Arimoto 1997: 


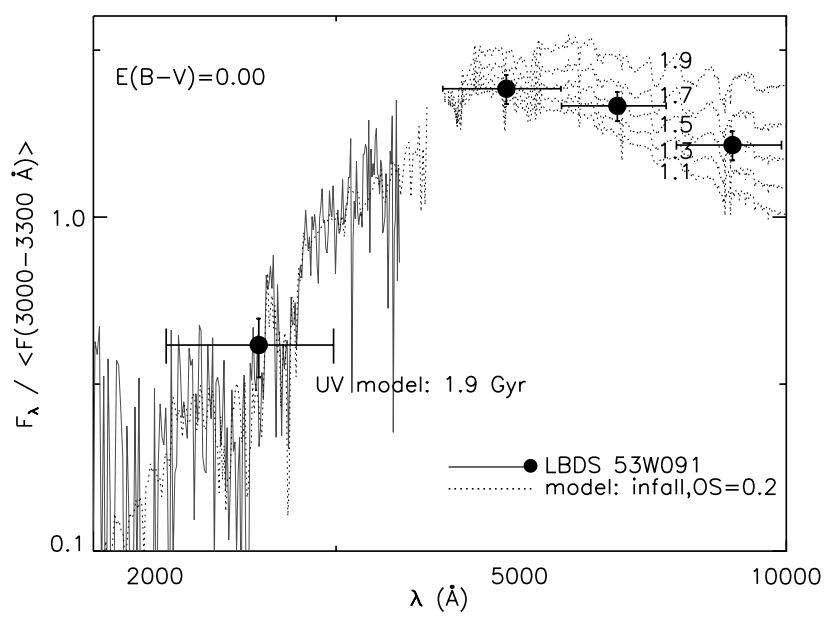

FIG. 12.-Model spectra compared to the observed data of LBDS 53W091. In the UV, only the best-matching model (with the minimum $\chi^{2}$ ) is overplotted, while in the longer wavelength range multiple models with various ages (shown in Gyr on lines) are shown. The UV spectrum indicates somewhat larger age than the the photometric data in the longer wavelength regions. This may be due to radial gradients in age and/or metallicity, or to reddening.

sample fit. The observed data are shown as a continuous line and filled circles. The overplotted models are those with the infall mixture and OS, which are probably more realistic than single-abundance models with no OS. In the UV, only the model with the minimum $\chi^{2}(1.9 \mathrm{Gyr})$ is shown for clarity. In the longer wavelength regions, multiple models with various ages are plotted over the photometric data. This figure demonstrates the difference between the age estimates from the analyses of the UV spectrum and of the photometric data.

This difference between the two age estimates may appear substantial to some readers. However, such a difference would not be unnatural if the data have been affected by some reddening, whether it is Galactic or internal. If we use the extinction curve of Cardelli, Clayton, \& Mathis (1989), in conjunction with that of O'Donnell (1994), a moderate amount of Galactic reddening, $E(B-V)=0.04$, reduces the difference between these two age estimates. In fact, the Galactic foreground extinction is estimated at $0.03 \mathrm{mag}$ by Schlegel, Finkbeiner, \& Davis (1998), along this line of sight $\quad\left(\right.$ R.A. $=17^{\mathrm{h}} 21^{\mathrm{m}} 17^{\mathrm{s}} 84, \quad$ decl. $=50^{\circ} 08^{\prime} 47^{\prime \prime} \cdot 7, \quad 1950.00$; $l=+76.8684, b=+34.4531)$. Then, both the UV spectrum and the photometric data become consistent with an age of $1.3 \mathrm{Gyr}$ (Fig. 13).

It is also probable that some of this difference comes from the fact that the light sources of the UV spectrum are older and/or more metal rich than those of the photometric data, because the UV spectroscopy covered a smaller area near the center of this galaxy (Spinrad et al. 1997). Then, composite models with radial gradients in age and metallicity would be more realistic to explain the observed data.

Our UV-based age estimates are in principle lower limits because the UV spectrum is likely to be dominated by the youngest population. However, if the star formation timescale was only an order of $0.1 \mathrm{Gyr}$, as many galactic chemical evolution models for giant elliptical galaxies suggest (e.g., Kodama \& Arimoto 1997), this age spread effect may not be large. It is important to note that our UV-based age estimates are larger than those from the photometric data. First, this may indicate the presence of at least some

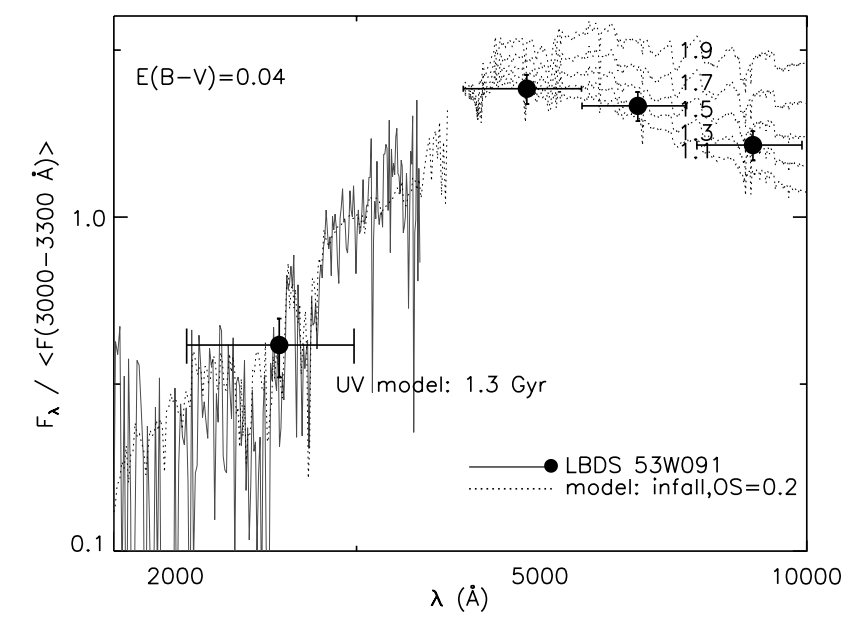

Fig. 13.-Same as Fig. 12, but with the effect of reddening included. The models are reddened according to the extinction curves of Cardelli et al. (1989) and of O'Donnell (1994). A 1.3 Gyr model matches both UV and visible-IR data reasonably.

reddening. We have shown above that a small amount of reddening reconciles the difference between the two age estimates. Second, if there is any substantial age spread in LBDS 53W091, the UV-based age estimates should be smaller than the visible-based ones, because the shorter wavelength spectrum is more dominated by the younger stars. For example, if a galaxy experiences a starburst that lasts for $1 \mathrm{Gyr}$ at a constant rate centered at $2 \mathrm{Gyr}$ before the observation, its integrated UV spectrum would be matched best by a single-burst model of an age of 1.8-1.9 Gyr instead of 2.0 Gyr. In some ad hoc star formation scenarios, the age underestimation can of course be larger. However, in the case of LBDS 53W091, UV-based age estimates are generally larger than the visible-based ones, which is opposite to the expectation from the population with any age spread. Thus, this likely implies very little age spread in LBDS 53W091 and the presence of some reddening, unless it is entirely due to the aperture effect. Then, our UV-based age estimates may even be an upper limit, rather than a lower limit. Without knowing the accurate amount of age spread and reddening and the level of accuracy of the models, it is not clear whether our estimates can constrain lower limits or upper limits.

\section{ORIGIN OF THE DISAGREEMENT AMONG VARIOUS MODELS}

The large difference in age estimate from Spinrad et al. and from this study is mainly due to the significant difference in the model integrated spectrum. Figure 14 shows the comparison between the latest Jimenez models ${ }^{1}$ (the preferred models in the analysis of Spinrad et al.) and the Yi models, both for the solar composition with no OS. The Jimenez models shown here are the new ones based on the stellar evolutionary tracks with finer mass grids and so probably improved over those used in Spinrad et al. (1997). At the time of this study, only his new models were made available to us.

In the UV, Jimenez's 1 Gyr model (1J) agrees with Yi's model (1Y) remarkably well. However, they substantially

\footnotetext{
May.
} 

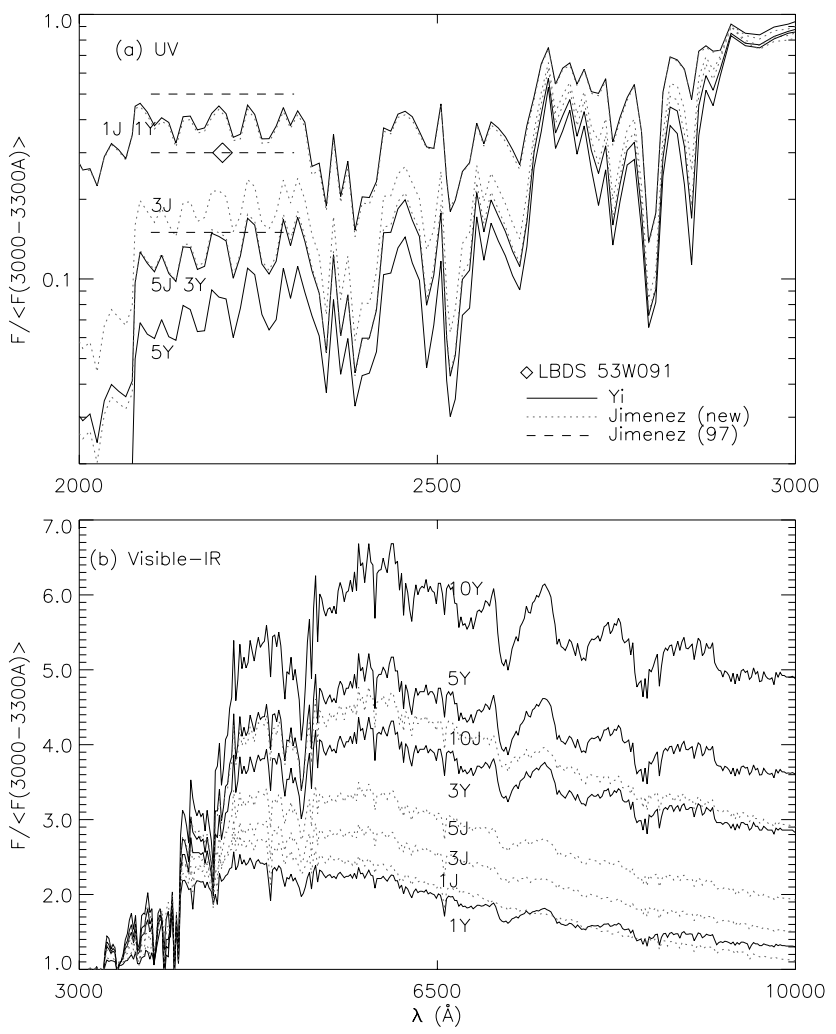

FIG. 14. - Comparison between the Yi models (marked "Y") and the Jimenez models (marked "J") for solar composition. Ages in Gyr are marked on each model. Both in the $(a)$ UVand in the $(b)$ visible-IR, the Jimenez models are substantially bluer.

disagree for larger ages: Yi's 2, 3, 5 Gyr models are close to Jimenez's 3, 5, 10 Gyr models (not all models shown here for clarity). In fact, Jimenez's $3 \mathrm{Gyr}$ and 5 Gyr models are nearly on top of Yi's 2 Gyr and 3 Gyr models. One can easily understand why Spinrad et al. and we are achieving such different age estimates. An open diamond in Figure $14 a$ is an approximate relative flux of LBDS 53W091 normalized at $3150 \AA$. This relative flux of LBDS 53W091 is closely reproduced by the $1.4 \mathrm{Gyr}$ model when the Yi models are used (see also Table 2) or the $1.9_{-0.1}^{+0.2}$ Gyr models when the new Jimenez models are used (based on the reduced- $\chi^{2}$ tests shown in eq. [2]).

Also plotted in Figure $14 a$ are the relative fluxes from the earlier version Jimenez models (horizontal dashed lines) used in Spinrad et al. (1997). Because we did not have access to this version Jimenez models, we read off the relative fluxes from Figure 14 of Spinrad et al. (1997). The three dashed lines are from the top his 1,3 , and 5 Gyr models, respectively. Note that his early version models are substantially bluer than his new version. One can see that the early version Jimenez models match the LBDS 53W091 data approximately at $3 \mathrm{Gyr}$, as Spinrad et al. (1997) suggested. The fit to the UV spectrum using the new Jimenez models, which suggests an age estimate of $1.9 \mathrm{Gyr}$, is shown in Figure 16a. Readers are encouraged to compare Figure $16 a$ to Figure 14 of Spinrad et al. (1997). As shown in Figure 15, the Yi models are somewhat redder than the 1999 version Bruzual \& Charlot (1999, private communication, hereafter BC) models, probably because the BC models assume a larger UV light contribution from post-asymptotic giant branch (PAGB) stars, but the overall agreement is good. If the new Jimenez models are improved over his previous
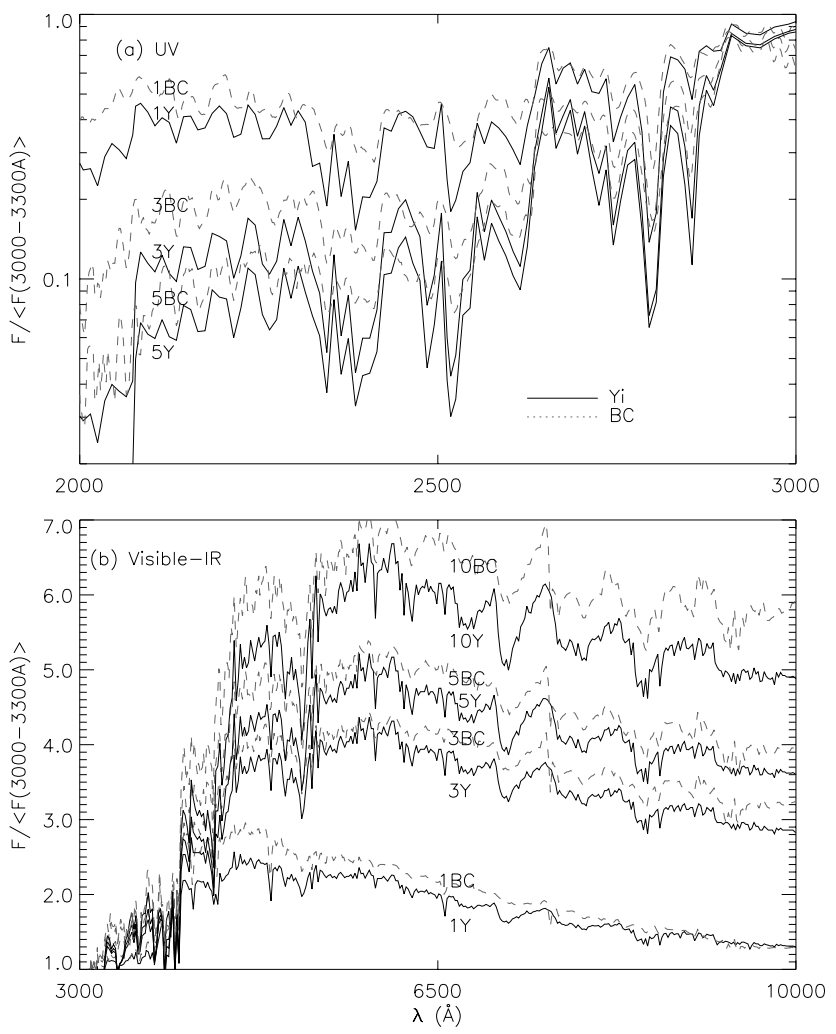

FIG. 15.-Comparison between the Yi models (marked "Y") and the 1999 version Bruzual \& Charlot models (marked "BC") for solar composition. They are in good agreement, except that the $\mathrm{BC}$ models are somewhat redder because they are based on the corrected Kurucz spectral library (see text).

version used by Spinrad et al., all three groups now suggest rather consistent age estimates, between 1 and 2 Gyr.

In the visible-IR, the difference between the new Jimenez models and the Yi models is even larger. Figure $14 b \mathrm{com}$ pares their 1, 3, 5, and $10 \mathrm{Gyr}$ models. Jimenez's 1,3 and 5 Gyr models are very close to one another, while Yi's models are quite well separated. Jimenez's 5 and $10 \mathrm{Gyr}$ models are quite close to Yi's 2 and 4 Gyr models, respectively. The Yi models are in close agreement with the $\mathrm{BC}$ models, as shown in Figure $15 b$. The BC models are based on a modified Kurucz spectral library (Lejeune, Cuisinier, \& Buser 1997) and thus are somewhat redder than the Yi models. The difference between the Jimenez models and the Yi models, however, is not the only cause for Spinrad et al. to get such a large age estimate, i.e., $2.5 \mathrm{Gyr}$, from their analysis on the photometric data. It was also caused by the fact that they used only $R-K$, omitting $J$ and $H$ magnitudes in their analysis. Figure $16 b$ shows that even the Jimenez models would have suggested a substantially smaller age if the whole photometric data had been used in their analysis. When we use the new Jimenez models and the reduced- $\chi^{2}$ test on the all of the four photometric data points, the best model indicates $1.9_{-0.7}^{+0.5} \mathrm{Gyr}$, which is in good agreement with the estimate from the UV analysis. Then again, all three groups scrutinized here are suggesting consistent age estimates of 1-2 Gyr. Such an agreement, at least on the age of LBDS 53W091, is possible because, for small ages $(\$ 2 \mathrm{Gyr})$, the new Jimenez models differ from the Yi and the BC models only slightly.

One may then say that the age discrepancy on LBDS 53W091 has been resolved. Despite this apparent 

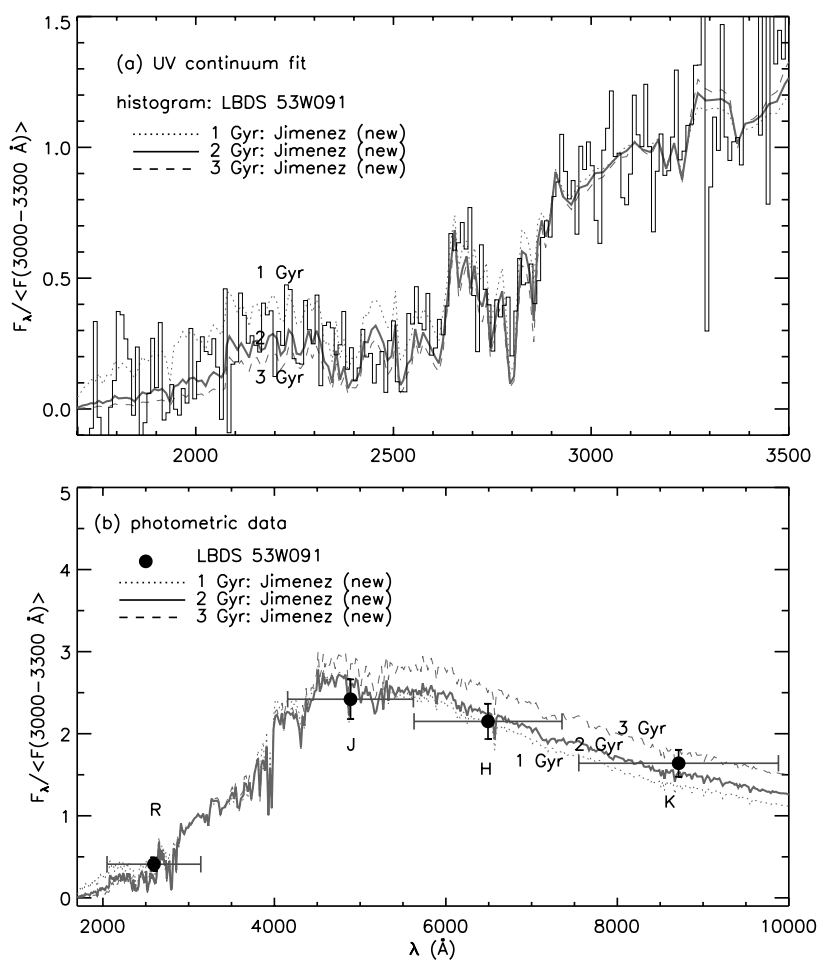

FIG. 16.-Fit to the data of LBDS 53W091 using the new Jimenez models. Both its UV spectrum and the photometric data consistently suggest approximately 1.9 Gyr.

resolution, it is still quite disturbing to know that there are significant disagreements between the Jimenez models and the Yi (and the BC) models at larger ages. If such a large disagreement is due to the uncertainties in the input physics, we would have to admit that we are not ready to estimate ages of the bulk of stellar populations via continuum fitting.

A notable disagreement can easily be introduced by the details in the population synthesis technique. For example, inaccurate interpolations between tracks, whether they take place during the isochrone construction or directly in the population synthesis, may cause significant difference especially in the visible-IR spectrum. The large impact of inaccurate mass interpolation has already been pointed out in $\S 2$ and perhaps demonstrated by the change in the two versions of the Jimenez models.

Despite such complexities in modeling the visible-IR flux, it is not true that model spectra in the visible-IR are generally unreliable (see Dunlop 1999 for a different opinion). For example, the age estimate using the old (1987) Revised Yale Isochrones, where RGB tips were computed less accurately than in the current Yale Isochrones used in this study, is different from our current estimate only by $20 \%$. The difference between the estimates of Bruzual \& Magris (1997a), based on the BC models, and ours is also small, even though the two groups use different stellar evolutionary tracks. Besides all these, the conventional population synthesis models beautifully match the overall spectra (near-UV through near-IR) of globular clusters (e.g., Bruzual et al. 1997) at their accepted ages. In short, the visible-IR flux is more sensitive to the uncertainties in the stellar models and in the population synthesis (including isochrone construction), but the level of uncertainty should be less than $20 \%$ or so in age. Thus, it is still unclear where such a large difference in the model spectrum as present between the Jimenez models and the Yi (and the BC) models comes from. It is unlikely to be caused by the difference in the input physics in the stellar models, because the stellar evolution theory is already quite well established. All these three groups use the Kurucz spectral library or some hybrid versions originated from it, and, thus, it is also unlikely that much of the difference can be attributed to the spectral library. The source of the discrepancy will be known only when these models are compared step by step with each other.

While it may be extremely difficult to directly compare different population models and find a more realistic model, it should be possible to test models by matching the observational properties of the objects whose ages are reasonably well known. Good examples include the Sun, M32, and Galactic globular clusters. We are presenting the results of the tests on the Sun and M32 only, because globular clusters have already been modeled successfully by a number of studies including that of Bruzual et al. (1997).

The following test results may not appear relevant to some readers, because the Sun and M32 are likely significantly older than LBDS 53W091. However, we would like to provide some useful sample tests that can easily be used to validate population synthesis models.

\subsection{Test on the Sun}

The age of the Sun, approximately $4.53 \pm 0.4 \mathrm{Gyr}$ (see Guenther \& Demarque 1997 for references), is one of the best constrained quantities in astronomy. In a 5 Gyr coeval population, most of the UV light is still produced by MS stars. Thus, a 5 Gyr old solar abundance population should exhibit a UV flux similar to that of the Sun. Figure 17 shows the fits to the theoretical solar spectrum $(2000-3500 \AA)$ from the Kurucz library using the Yi models (panel a) and the new Jimenez models (panel b). Our test on the Sun is not weighted with observational errors because the solar spectrum is a theoretical one. In this sense, we are measuring merely the mean square difference (MSD) between the model and the data instead of $\chi^{2}$. The formula for the MSD test is shown in equation (3):

$$
\operatorname{MSD}=\frac{1}{N-2} \sum \frac{\left[f_{\lambda}(\text { model })-f_{\lambda}(\text { object })\right]^{2}}{f_{\lambda}(\text { object })},
$$

where $N$ is the number of spectral bins.

The best-fitting model is a $5.0 \pm 0.1 \mathrm{Gyr}$ model when the Yi models are used. This slight disagreement in the age estimate from the generally accepted solar age is perfectly expected, because we are matching a single stellar spectrum with those of composite (in the sense of containing MS, red giants, etc.) stellar populations and the light contribution in the UV from post-MS stars (mostly from PAGB stars) is slightly larger in the far-UV than in the near-UV. The proximity of this age estimate to the accepted age of the Sun once again demonstrates the high reliability of the UVbased age estimates for intermediate-age, composite populations. However, when the new Jimenez models are used, much larger ages are indicated (10 Gyr giving the best fit). This was already evident in Figure 14, where the Jimenez models appeared much bluer than the Yi models. If the continua of G-type stars (including the Sun) in the Kurucz library are significantly inaccurate, an effort to achieve the right age of the Sun using population synthesis models and a theoretical spectrum would not be appropriate. Yet, such 


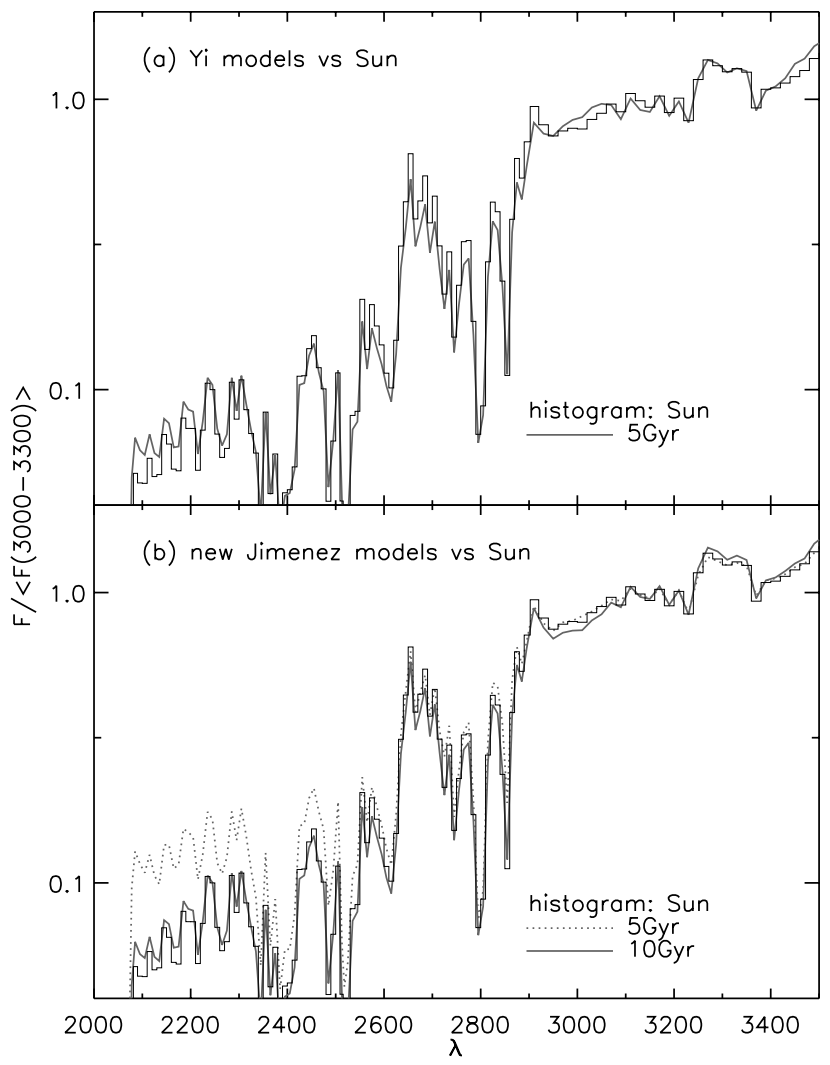

FIG. 17.-Test of the Yi (top) and new Jimenez (bottom) population synthesis models on the Sun. Models are composite in the sense that they contain all evolutionary phases. But, at $5 \mathrm{Gyr}$, MS stars still dominate the UV spectrum ( $92 \%$ at $2200 \AA, 96 \%$ at $3150 \AA)$; thus, the composite UV spectrum model should match the solar spectrum at a reasonably close age to the known one, i.e., 4.5-4.7 Gyr.

tests would still serve as sanity checks for the population synthesis computations.

\subsection{Test on $M 32$}

M32 also provides a good test, as we now have resolved visible-IR CMDs that suggest an age of $\approx 8.5 \mathrm{Gyr}$ (Grillmair et al. 1996). We have adopted the visible-IR spectrum of M32 from Bruzual \& Magris (1997b). Figure 18 shows the fits using the solar abundance models of Yi and of Jimenez (new). Both the Yi models and the Jimenez models are based on the theoretical Kurucz spectral library, and thus their fits are limited by the known shortcoming of the Kurucz library in matching the spectra of cool stars. In addition, these single-age, single-abundance models may not be good approximations to M32. We find that the match becomes better, in particular in the $U$ band and in the IR, when chemically composite models are used. Bearing all these limitations in mind, we cautiously find that the Yi models match the M32 spectrum somewhat better.

In conclusion, we note the following. The difference in the age estimate between Spinrad et al. (1997) and this study is caused by the large difference in the model integrated spectrum. The Jimenez models, their preferred models, appear much bluer than the $\mathrm{Yi}$ and the $\mathrm{BC}$ models. The new Jimenez models, presumably improved over his earlier version, are redder than his previous models and thus closer to the $\mathrm{Yi}$ and the $\mathrm{BC}$ models. However, they are still much bluer than the Yi and the $\mathrm{BC}$ models. Currently, the Yi models are in reasonable agreement with the $\mathrm{BC}$ models

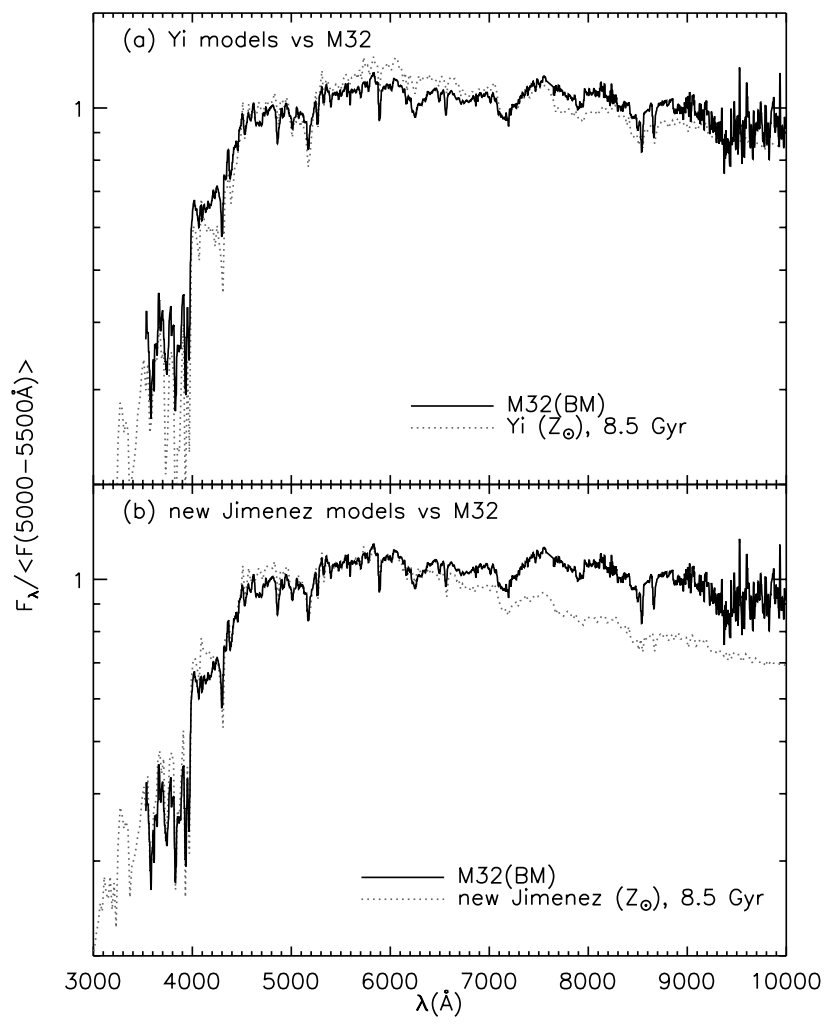

FIG. 18.- Test of the $(a) \mathrm{Yi}$ and new Jimenez $(b)$ population synthesis models on M32 using its visible-near IR spectrum from Bruzual \& Margris (1997b). Models are for the solar chemical composition and for the age of $8.5 \mathrm{Gyr}$, which has been determined from visible-near-IR CMD studies (see text). The new Jimenez models deviate from the observed spectrum.

and seem to match the spectra of the Sun and M32 at their accepted ages better than the new Jimenez models do.

\section{SUMMARY AND CONCLUSION}

The pioneering studies of Spinrad and his collaborators (Dunlop et al. 1996; Spinrad et al. 1997) have demonstrated the significance of precise age estimates of distant galaxies. Their suggestion that the red galaxy, LBDS 53W091, at $z=1.552$ is at least $3.5 \mathrm{Gyr}$ old was striking because it would result in a rather strict constraint on cosmology. We have carried out a similar exercise, estimating the age of this galaxy, but only via continuum fitting. When we use the same input parameters, our age estimate is approximately 1.4-1.8 Gyr, substantially smaller than theirs, but consistent with those of Bruzual \& Magris (1997a) and of Heap et al. (1998).

The large age estimate of Spinrad et al. is apparently caused by the use of the early Jimenez models in their analysis, which are significantly bluer than the Yi and the BC models. The latest Jimenez models are somewhat redder than his earlier models and closer to the Yi models, resulting in the age estimates that are consistent with our estimates. This may indicate a resolution of the age discrepancy on LBDS 53W091.

We have further improved our estimates over previous ones by adopting convective core overshoot (OS) and realistic metallicity mixtures. The inclusion of OS has little effect on the UV-based age estimates, but it raises the age estimates based on the visible data normalized to the UV by $20 \%-50 \%$. Adopting realistic metallicity distributions is 
also important because different metallicity groups dominate different parts of the integrated spectrum. If we assume that the majority of stars in LBDS 53W091 are already as metal rich as those in nearby giant elliptical galaxies, the photometric data of LBDS 53W091 indicate up to a factor of 2 larger ages when metallicity mixtures are adopted.

The UV continua of young galaxies, such as LBDS 53W091, are not sensitive to OS. In addition, solar abundance models reasonably approximate them. This relative immunity of the UV data against such complexities makes the UV spectrum of a distant galaxy a very useful age indicator. Our UV-based estimate is approximately $2.0 \pm 0.2$ Gyr and apparently inconsistent with that of Spinrad et al., $3.5 \mathrm{Gyr}$, but consistent with the age estimates we obtained using the new Jimenez models.

The photometric data of LBDS 53W091 indicate $1.5 \pm 0.2$ Gyr. The slightly larger estimates from the UV continuum fit would be consistent with this photometrybased one if we include a small amount of reddening and/or if the core of this galaxy is somewhat older or more metal rich than its outskirt, all of which are quite plausible. It may also indicate that there is no substantial age spread among the stars in LBDS 53W091.

The age estimates of Spinrad and his collaborators were heavily based on selected UV spectral breaks. This was because UV spectral breaks were believed to be less sensitive to the uncertainties in reddening. If this is true and if we are ignoring the possible reddening effects, our age estimates should be systematically larger than theirs because reddening makes the continuum look older, which is opposite to what we have found. Thus the difference between Spinrad et al.'s estimate and ours cannot be reconciled by adopting any conventional reddening law.

Our results on LBDS 53W091 are vulnerable to the uncertainties in the spectral library in matching the F-type stellar spectra in the UV. Such uncertainties may exist not only in the detailed spectral features, such as those studied by Spinrad et al. (1997) and Heap et al. (1998), but also in the continuum. The same authors of Heap et al. (1998) are currently obtaining the UV spectra of F-type stars using HST/STIS. When the project is complete, a more accurate analysis both on the spectral breaks and the continuum will be possible.

There is no doubt that precise age estimates of high- $z$ galaxies would be very useful for constraining cosmology. In order to fully take advantage of the power of this technique, however, we first need to understand the details of the population synthesis, which are currently creating a substantial disagreement in age estimate. We propose to carry out a comprehensive investigation on the various population synthesis models through a series of standard tests on the objects whose ages have been independently determined. Such objects may include the Sun, M32, and Galactic globular clusters. Our models (the Yi models) currently pass these tests reasonably.

Our age estimates indicate that LBDS 53W091 formed approximately at $z=2-3$. However, our smaller age estimate for this one galaxy does not contradict work that suggests galaxies generally formed at high redshifts, regardless of the rarity of massive elliptical galaxies at $z \approx 1.5$. Furthermore, we are just beginning to expand our observations of galaxies to high redshift, and so the existence of a few old galaxies at high redshifts does yet prove any galaxy formation scenario, although it can potentially constrain cosmological parameters (in the sense that the ages of a few objects can provide lower limits on the age of the universe at that redshift). Finding no old galaxies at high redshift would support a low $z_{f}$ for the general population. Building a larger database of observations is therefore crucial to achieve a unique and statistically significant solution. Dunlop (1999) reported a discovery of another galaxy (LBDS 53W069) whose UV spectrum looks even redder than that of LBDS 53W091, although its redshift is only slightly smaller $(z=1.43)$. This would be a stronger sign of large ages of high- $z$ galaxies. As more data are collected, our vision to the high- $z$ universe will be clearer.

This work was encouraged by the open-minded response of Hyron Spinrad to our initial interest in the work on LBDS 53W091 done by him and his collaborators. We thank his group, in particular Daniel Stern, for providing the spectrum of LBDS 53W091. We are grateful to Taddy Kodama for providing his metallicity distribution models and to Gustavo Bruzual for providing the spectrum of M32. The constructive criticisms and comments of Raul Jimenez, Hyron Spinrad, Gustavo Bruzual, Sydney Barnes, and Pierre Demarque improved the manuscript significantly. We owe special thanks to Raul Jimenez and Gustavo Bruzual for making their models available to us. This work was supported by the Creative Research Initiative Program of the Korean Ministry of Science and Technology grant. Part of this work was performed while S. Y. held a National Research Council-(NASA Goddard Space Flight Center) Research Associateship.

\section{REFERENCES}

Aldering, G., et al. 1998, A\&AS, 193, 3904

Angeletti, L., \& Gianonne, P. 1999, A\&A, 343, 720

Bruzual A., G., Barbuy, B., Ortolani, S., Bica, E., Cuisinier, F., Lejeune, T., \& Schiavon, R. P. 1997, AJ, 114, 1531

Bruzual A., G., \& Magris, G. C. 1997a, in AIP Conf. Proc. 408, The Ultraviolet Universe at Low and High Redshift, ed. W. H. Waller (Woodbury: AIP), 291

. 1997b, poster presented at 1997 STScI May Symp. on Hubble Deep Field (astro-ph/9707154)

Cardelli, J. A., Clayton, G. C., \& Mathis, J. S. 1989, ApJ, 345, 245

Demarque, P., Sarajedini, A., \& Guo, X.-J. 1994, ApJ, 426, 165

Dunlop, J. 1999, in The Most Distant Radio Galaxies, ed. P. N. Best, H. J. A. Röttgering, \& M. D. Lehnert (KNAW Colloq.; Amsterdam: Kluwer), 71

Dunlop, J., Peacock, J., Spinrad, H., Dey, A., Jimenez, R., Stern, D., \& Windhorst, R. 1996, Nature, 381, 581

Green, E. M., Demarque, P., \& King, C. R. 1987, The Revised Yale Isochrones and Luminosity Functions (New Haven: Yale Univ. Obs.)

Grillmair, C. J., et al. 1996, AJ, 112, 1975

Guenther, D., \& Demarque, P. 1997, ApJ, 484, 937
Heap, S. R., et al. 1998, ApJ, 492, L131

Kodama, T., \& Arimoto, N. 1997, A\&A, 320, 41

Kozhurina-Platais, V., Demarque, P., Platais, I., Orosz, J. A., \& Barnes, S. 1997, AJ, 113, 1045

Kurucz, R. 1992, in The Stellar Population in Galaxies, ed. B. Barbuy \& A. Renzini (Dordrecht: Reidel), 225

Lejeune, Th., Cuisinier, F., \& Buser, R. 1997, A\&AS, 125, 229

O'Donnell, J. E. 1994, ApJ, 422, 158

Rogers, F. J., \& Iglesias, C. A. 1992, ApJS, 79, 507

Schlegel, D., Finkbeiner, D. P., \& Davis, M. 1998, ApJ, 500, 525

Schwarzschild, K. 1906, Göttingen Nachr., 41

Shaviv, G., \& Salpeter, E. 1973, ApJ, 184, 191

Spinrad, H., Dey, A., Stern, D., Dunlop, J., Peacock, J., Jimenez, R., \& Windhorst, R. 1997, ApJ, 484, 581

Stothers, R. B. 1991, ApJ, 383, 820

Yi, S., Demarque, P., \& Kim, Y.-C. 1997a, ApJ, 482, 677

Yi, S., Demarque, P., \& Oemler, A., Jr. 1997b, ApJ, 486, 201

Yi, S., Lee, Y.-W., Woo, J.-H., Park, J.-H., Demarque, P., \& Oemler, A., Jr. 1999, ApJ, 513, 128 